\title{
Urologic Tissue Engineering in Pediatrics: From Nanostructures to Bladders
}

\author{
CHRISTOPHER C. ROTH \\ Department of Urology, Louisiana State University Health Sciences Center, New Orleans, Louisiana 70118
}

\begin{abstract}
The use of tissue engineering to develop new urinary bladder could have direct impact on children suffering from congenital bladder dysfunction. Current bladder tissue engineering protocols rely on scaffolds to support the ingrowth of new cells and the subsequent development of new tissues. The interaction between cells and scaffolds is mediated on the molecular level and nanotechnology is being investigated as a means to improve the biocompatibility of these scaffolds. In this review, we will discuss the current applications of nanotechnology for bladder tissue engineering; specifically, the use of selective degradation techniques and selfassembly molecules to create nanofeatures on existing scaffolds, the use of electrospinning to create de novo scaffolds, and the use of nanoparticles as a drug delivery system will be discussed. The use of nanotechnology has resulted in novel tissue engineering protocols and is likely to be an important factor in the eventual clinical application of tissue engineering for the urinary bladder. (Pediatr Res 67: 509-513, 2010)
\end{abstract}

$\mathrm{T}_{\mathrm{s}}^{\mathrm{b}}$ he pediatric population is susceptible to several congenital anomalies that can affect overall urinary bladder function. Children with neurogenic bladder, bladder outlet obstruction, and bladder exstrophy are at risk for poor bladder function and may eventually require bladder replacement therapies. Traditional bladder replacement therapies often involve the use of enteric segments to either replace or augment the bladder. The incorporation of enteric segments into the urinary tract caries potential metabolic, infectious, and neoplastic risk, a consideration that has encouraged researchers to seek an alternative source of donor tissue.

The field of tissue engineering involves identifying pathways of tissue regeneration and promoting these pathways by providing the raw materials needed by the body to restore the original design and function of pathologic tissue. Most tissue engineering strategies involve the use of a scaffold or matrix to guide the structural development of new tissues. Some protocols include seeding cultured cells from the organ of interest onto the graft to promote regeneration; other protocols simply use the grafts alone with dependence on the organism to provide the cells needed for the developing tissue. The potential therapeutic benefits of tissue engineering in urology are numerous; similarly, interest in tissue engineering among researchers and clinicians is rapidly expanding. Despite the

Received November 3, 2009; accepted November 24, 2009.

Correspondence: Christopher C. Roth, M.D., Department of Urology, Louisiana State University Health Sciences Center, 200 Henry Clay Avenue, New Orleans, LA 70118; e-mail: croth@lsuhsc.edu remarkable amount of basic science research, there has been limited success in clinical application of this technology.

Nanotechnology is currently being explored as a means to improve existing bladder tissue engineering protocols. Nanotechnology is a broad term, which can be defined as creation of objects or surfaces whose unique functions are a direct result of their nanoscale dimensions and/or organization (1). Tissue engineering relies on interactions between cells and their substrates and these interactions, often mediated by cell receptors and ligands, are carried out on the nanoscale level. An understanding of molecular biology allows researchers to design substrates with specific nanofeatures in an effort to improve cell-substrate interactions. In regard to bladder tissue engineering, nanotechnology has been used to develop novel biomaterials through both enhancement of existing scaffolds and creation of de novo nanoscale substrates. This review will outline the current applications of nanotechnology for bladder tissue engineering; specifically, the use of selective degradation techniques and self-assembly molecules to create nanofeatures on existing substrates, the use of electrospinning to create de novo substrates, and the use of nanoparticles (NPs) as a drug delivery system will be discussed.

\section{NANOSCALE MODIFICATION OF EXISTING SUBSTRATES}

Scaffolds used in tissue engineering protocols can be broadly classified as natural and synthetic. Natural scaffolds, for example extracellular matrices, contain ubiquitous molecular compounds such as collagen and fibronectin which promote cell adhesion. The use of natural scaffolds is limited by their heterogeneous nature and limited natural supply. Synthetic scaffolds are commonly polymers or co-polymers of inert, biodegradable compounds which can be produced in large quantities and with varying physical properties. Synthetic scaffolds lack natural ligands for cell adhesion and nanotechnology is being investigated as a means to enhance cell-substrate interactions and biocompatibility.

Natural extracellular matrices are composed of nanoscale proteins, which promote cellular adhesion by both their chemical and physical properties. Chemical etching and selective

Abbreviations: HA, hyaluronic acid; NP, nanoparticle; PA, peptide-amphiphile; PCL, poly-caprolactone; PEUU, poly (ester-urethane) urea; PLGA, poly-lactic-co-glycolic acid; PU, poly-ether-urethane; SIS, small intestinal submucosa; UBM, urinary bladder matrix 
degradation techniques can be used to create nanoscale physical features on existing synthetic substrates with the hope of improving cell adhesion and ingrowth (2). Research at Purdue University under the direction of Haberstroh has examined the use of selective degradation of synthetic scaffolds to influence bladder smooth muscle adhesion. Their initial work involved modification of three commonly used polymers, poly-lacticco-glycolic acid (PLGA), poly-ether-urethane (PU), and polycaprolactone (PCL) (3). $\mathrm{NaOH}$ (for PLGA, PCL) and $\mathrm{HNO}_{3}$ (for PU) were used at varying concentrations to selectively degrade the surface features of the scaffold. Scanning electron microscopy was used to assess the topographic features of the polymers and demonstrated that treating PLGA scaffolds with $10 N$ of $\mathrm{NaOH}$ for 1 hour, PU scaffolds with $10 \mathrm{~N}$ of $\mathrm{HNO}_{3}$ for 30 min, and PCL scaffolds with $0.1 \mathrm{~N}$ of $\mathrm{NaOH}$ for 10 min resulted in reduction of surface feature dimensions into the nanometer range (Fig. 1). Bladder smooth muscle cells were seeded onto the experimental substrates and cell adhesion was analyzed at varying time points. Fluorescent microscopy demonstrated statistically significant improved cellular adhesion in the modified nanostructured scaffolds (surface features 50-100 nm) compared with unmodified scaffolds (surface features 10-15 $\mu \mathrm{m}$ ).

In the earlier study, it was also noted that chemical degradation of the polymers resulted in altered surface chemistry of the scaffolds. In an important follow-up study, inert molds were used to create scaffolds with the same nanostructured features though without an alteration in surface chemistry. In vitro experiments noted improved smooth muscle cell adhesion in mold-derived scaffolds indicating that the change in size and not the change in chemical features was responsible for improved cell adhesion (4).

The same group of researchers performed additional in vitro work to further characterize protein production by cultured cells on nanostructured PLGA and to determine what effect hydrostatic pressure would have on cell growth. Again, improved cell adhesion was noted on the nanostructured scaffolds with an additional finding of increased collagen and elastin production within these grafts. At a pressure designed to mimic in vivo bladder pressure, $10 \mathrm{~cm} \mathrm{H}_{2} \mathrm{O}$, no difference in cell growth or protein production was noted when compared with scaffolds at atmospheric pressure (5).

The authors hypothesized that the nanoscale features of the modified scaffolds may yield improved protein adsorption and thus improved cell adhesion and growth. This hypothesis was tested by comparing fibronectin and vitronectin (proteins im-
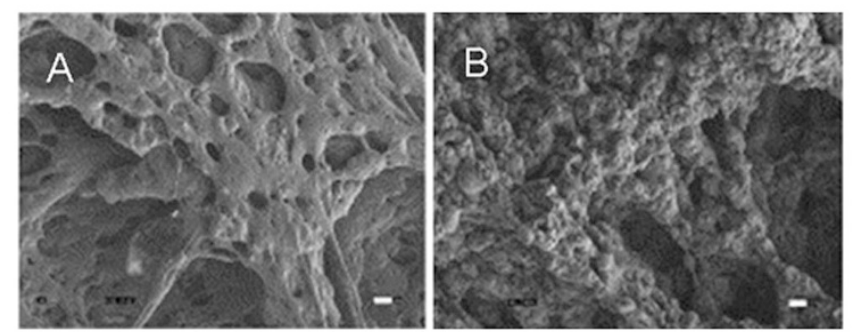

Figure 1. Representative scanning electron micrographs of PLGA. Image of conventional PLGA $(A)$ and nano-PLGA $(B)$ at $2500 \times$ magnification. Scale bar $=10 \mu \mathrm{m}$. Reprinted from Pattison et al. 2005 Biomaterials 26:24912500. Copyright @ 2004 Elsevier Ltd., with permission. portant for cellular adhesion) adsorption on conventional and nanostructured PLGA. The nanostructured PLGA demonstrated increased adsorption of both proteins when analyzed using ELISA. Endothelial and vascular smooth muscle cell growth was improved on nanostructured PLGA versus conventional PLGA when both types of scaffolds were preadsorbed with vitronectin and fibronectin. The authors concluded that enhanced protein adsorption by the nanostructured PLGA may contribute to improved cell adhesion to these scaffolds (6).

The most recent study available from the Purdue group performed additional in vitro work and initial in vivo experiments using the nanostructured scaffolds. Bladder smooth muscle cell adhesion and growth were again found to be greater on nanostructured PLGA and PU than conventional scaffolds with cell adhesion being the greatest for nanostructured PLGA. Collagen and elastin production were greater for cells grown on the nanostructured scaffolds when compared with conventional scaffolds. A limited animal experiment was conducted using conventional 50:50\% PLGA scaffolds, nanostructured 50:50\% PLGA scaffolds, nanostructured 75:25\% PLGA scaffolds, nanostructured 85:15\% PLGA scaffolds, and nanostructured PU scaffolds. A $1 \times 1 \mathrm{~cm}^{2}$ area of rat bladder was removed and was replaced with one of the experimental scaffolds and the rats were killed at varying time points. Three of the four rats augmented with 50:50\% PLGA survived until they were killed at $11 \mathrm{mo}$ and the scaffold was found to be replaced by regenerated bladder. Two of three animals augmented with 50:50\% nanostructured PLGA developed bladder leaks and died before bladder harvest. Bladder regeneration was noted in the one remaining animal at the time of harvest. 75:25\% nanostructured PLGA was formulated to create a stronger graft with the hope of slower degradation and fewer bladder leaks. One of four animals augmented with 75:25\% nanostructured PLGA died of a bladder leak though bladder regeneration was noted at the time of harvest in the other three animals. The $85: 15 \%$ nanostructured PLGA was too brittle to suture and an augmentation could not be performed with this scaffold. In all of the animals augmented with nanostructured $\mathrm{PU}$, the grafts were found to be extruded either internally or externally to the bladder wall. Despite limited degradation of the graft, the authors identified regenerated bladder tissue in all of the PU animals (7).

This series of studies illustrates the benefit of nanoscale features in improving cell-substrate interactions and helps further the understanding of cell adhesion and growth on a molecular level. Additionally, the preliminary animal study simultaneously demonstrates the limitations and benefits of synthetic scaffolds. The lack of degradation of any synthetic scaffold, like that demonstrated by the PU scaffolds in the above study, is likely to limit their use in any large-scale model of regeneration. However, the ability to modify the composition of the PLGA scaffold to create a more durable graft illustrates the adaptability of synthetic scaffolds and underscores the reason why research into synthetic scaffolds will continue.

The earlier studies represent a "top-down" approach to fabrication of biomaterials, for example, the degradation of polymers to achieve surface features with nanoscale dimensions. Other researchers use a "bottom-up" approach, which 
uses individual molecules to self-assemble into larger objects with nanoscale dimensions. In self-assembly systems, specific features of the component molecules are selected to direct the eventual the shape and size of the aggregate molecule. The component molecules will often contain oppositional features (e.g. hydrophobic and hydrophilic, flexible and rigid), which force the component molecules to reduce their entropic/ enthalpic balance through aggregation. Peptide-based selfassembly systems have been investigated for their use as scaffolds in tissue engineering protocols (1).

Research at Northwestern University under Stupp has investigated the use of self-assembling peptide amphiphile (PA) molecules to enhance the biocompatibility of PLGA scaffolds (8). The PA molecules can be triggered to assemble into nanofibers with the biologically active peptide headgroups displayed along the fiber periphery. The PA systems can be engineered with varying peptide headgroups, and in this particular study, the amino acid sequence RGDS (arginine, glycine, aspartic acid, and serine) was chosen for its previously established role in mediating cell adhesion. The RGDS peptide can be presented in a linear form or through the incorporation of a branched l-lysine dendron, in a branched form. The aims of this particular study were to determine whether the PA system would self-assemble on the surface of PLGA and if linear or branched PA improved bladder smooth muscle cell adhesion compared with conventional PLGA.

They determined that the PA system would self-assemble on the PLGA and that PA molecules remained bound in their self-assembled form on the PLGA in cell culture conditions. Bladder smooth muscle cells were cultured on linear PA, branched PA, and bare PLGA. Cell adhesion and ingrowth were evaluated via scanning electron and light microscopy at 4, 10, 17, and $31 \mathrm{~d}$. At 4 and $10 \mathrm{~d}$, the branched PA scaffolds demonstrated improved cell growth and matrix deposition. At $17 \mathrm{~d}$, all groups demonstrated some cell coverage though the branched PA scaffold formed a monolayer of phenotypically mature cells. At $31 \mathrm{~d}$, there was no discernable difference between the groups. This study nicely demonstrates the application of nanotechnology to improve an existing tissue engineering protocol (seeding cells on PLGA). Tissue engineering can be viewed as race between scarring and regeneration and earlier cell adhesion and matrix deposition are likely to favor regeneration. In vivo studies will be needed to bare this out.

\section{DE NOVO CREATION OF NANOSCALE SCAFFOLDS VIA ELECTROSPINNING}

Electrospinning has recently gained popularity among researchers attempting to develop improved scaffolds for bladder tissue engineering. Electrospinning has obvious advantages in tissue engineering in that it offers an inexpensive and simplistic means to create nanoscale fibers from a large array of natural and synthetic polymers. The basic electrospinning apparatus consists of a syringe pump, a high voltage source, and a collector (Fig. 2). The polymer solution is held within the needle tip of the syringe by the surface tension of the solution. The high voltage source is used to create electrostatic forces within the polymer solution. Once the electrostatic

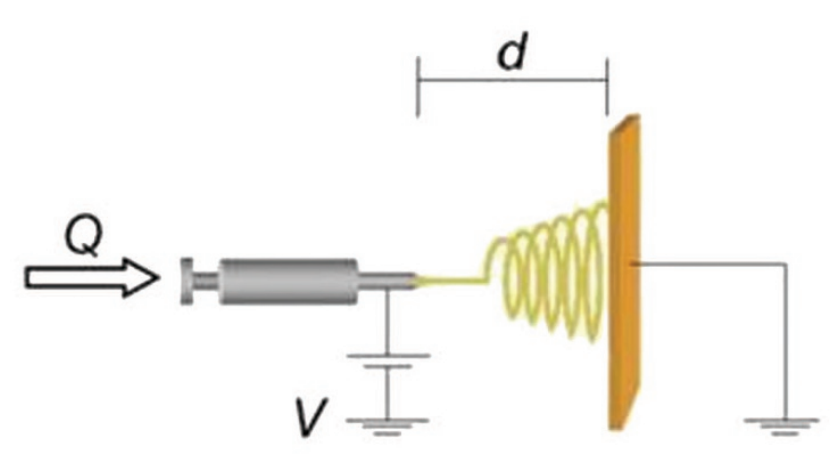

Figure 2. Schematic of electrospinning apparatus. $Q=$ flow rate, $d=$ distance between plate and needle, $\mathrm{V}=$ applied voltage. Manipulation of these variables results in qualitative changes in the resultant nanofibers. Reprinted from Pham et al. 2006 Tissue Eng 12:1197-1211. Copyright (C) 2006 Mary Ann Liebert Inc., with permission.

force overcomes the surface tension, a jet of polymer is extruded from the needle tip. Any solvent within the solution will evaporate while the polymer is in the jet form and before the polymer contacting the collector. The collector can be static or programmed to move in any plane (including spinning) to affect the eventual orientation of the fibers. Similarly, any of the other variables within the process, for example the composition of the polymer, the distance between the needle tip and collector, the solvent, and the voltage, can be adjusted to produce specific qualities within the electrospun fibers (9).

Han et al. have evaluated the use of electrospinning to create a scaffold modeled after bladder extracellular matrix (10). Scanning electron micrographs of bladder extracellular matrix demonstrated a tri-layered architecture consisting of a dense base layer, an intermediate cellular level with large pores from the ingrowth of cells, and a superficial fibrous layer. The authors were able to modify the electrospinning process to create a cellulose acetate scaffold with remarkably similar physical characteristics. Their testing demonstrated that dense scaffold could be produced by a low concentration of polymer solution, low flow rate, and high-voltage electrical field; therefore, the base layer of the scaffold was produced from a $7.5 \% \mathrm{wt} / \mathrm{vol}$ solution of cellulose acetate at a flow rate of $30 \mu \mathrm{L} / \mathrm{min}$ and filed strength of $19 \mathrm{kV}$. Removable copper wire coils with a diameter of $300 \mu \mathrm{m}$ were used to make a template, which was placed on top of the base layer. This template helped create the large pores within the cellular layer which was produced with $10 \% \mathrm{wt} / \mathrm{vol}$ solution and all other parameters left unchanged. The superficial fibrous layer was then added to the scaffold at a solution concentration of $17.5 \%$ $\mathrm{wt} / \mathrm{vol}$, flow rate of $160 \mu \mathrm{L} / \mathrm{min}$, and field strength of $7 \mathrm{kV}$. Scanning electron microscopy of the engineered scaffolds demonstrated remarkably similar characteristics to the native bladder extracellular matrix.

Additional studies have investigated the mechanical properties and in vitro biocompatibility of electrospun scaffolds. Baker et al. (11) used polystyrene to create electrospun scaffolds with varying spatial configurations. Scaffolds with transversely and longitudinally aligned fibers where compared with scaffolds with randomly oriented fibers and native bladder tissue (Fig. 3). In regards to ultimate stress, the scaffolds with 


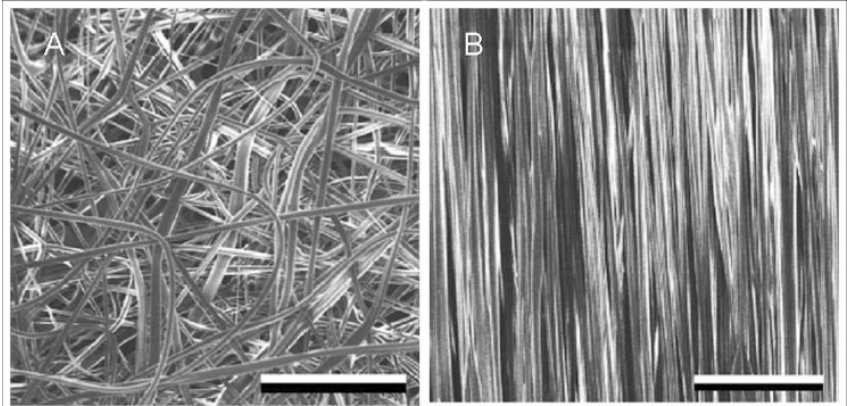

Figure 3. Representative scanning electron micrographs of electrospun polystyrene scaffold. Randomly aligned nanofibers $(A)$ and longitudinally aligned nanofibers $(B)$ created by increasing the rotation speed of the collecting mandrel. Scale bar $=20 \mu \mathrm{m}$. Reprinted from Baker et al. 2006 Biomaterials 27:3136-3146. Copyright (C) 2006 Elsevier Ltd., with permission.

longitudinally aligned fibers demonstrated similar strength to native bladder tissue and greater strength than transversely aligned and randomly aligned scaffolds. All of the scaffold materials had sufficient physical properties for use as a cellculture substrate. The effect of fiber alignment on cell growth of human bladder smooth muscle cells was evaluated. Confocal microscopy demonstrated that cells remained viable on both aligned and random scaffolds; however, staining for actin microfilaments demonstrated that cell alignment was greater when grown on scaffolds with aligned fibers. The authors concluded that characteristics of the scaffold could be configured to affect the phenotype of cultured cells.

Electrospun scaffolds can be constructed from natural materials and a combination of natural and synthetic materials. McManus et al. (12) evaluated bladder smooth cell proliferation on electrospun fibrinogen scaffolds. Early evaluation noted improved cell migration which later yielded improved proliferation and collagen deposition in the experimental fibrinogen scaffolds. Stankus et al. (13) evaluated producing scaffolds from a combination of biodegradable poly (esterurethane) urea (PEUU) and soluble urinary bladder matrix (UBM). A UBM solution was formulated by lyophilizing UBM, crushing the UBM into a powder, digesting the UBM powder with pepsin, and dissolving the UBM in 1,1,1,3,3,3,-hexafluoro-2propanol. Scaffolds with various ratios of UBM:PEUU were constructed. Scaffolds composed of UBM alone were too weak to support cell growth. Increasing the amount of PEUU resulted in improved mechanical properties. In vitro biocompatibility was assessed via vascular smooth muscle cell culture and findings demonstrated improved cell proliferation on scaffolds with higher ratios of UBM:PEUU. Subcutaneous scaffold implantation in mice revealed improved cell infiltration in the composite scaffolds when compared with PEUU scaffolds.

A modification of electrospinning has been developed to introduce cells directly into a de novo scaffold. As previously mentioned, some tissue engineering techniques rely on the creation of tissue constructs which consist of scaffolds plus cultured cells. Traditional cell seeding methods can be limited by the number of cell types that can be grown on given scaffold. Fullhase et al. have presented research, which allows them to incorporate three distinct cell lines into an electrospun scaffold (Fullhase C et al., A novel hybrid printing system for the generation of organized bladder tissue, 2009 American Urologic Association Annual Meeting, April 25-30, Chicago, IL, Abstract 791). The authors used an electrospinning apparatus to create PCL scaffolds. During the creation of the scaffold, a modified inkjet bioprinter was used to introduce the desired cell line at a specific location of the scaffold. With the goal of creating a trilayered bladder construct, urothelial cells were introduce on the luminal surface, lamina propria cells in the intermediate layer, and smooth muscle cells on the adluminal surface. These constructs were implanted into the flanks of mice and histologic evaluation after surgical retrieval demonstrated vascularized tissue consisting of three distinct cell layers.

The use of electrospinning to create nanofiber-based scaffolds holds promise for the development of novel biomaterials for use in bladder tissue engineering. The adaptability of the synthetic polymers and the ability to incorporate both natural biomaterials and cultured cells directly into the scaffold offer the opportunity to use electrospinning to construct highly organized and functional scaffolds.

\section{USE OF NANOSCALE POLYMERS AS A DRUG DELIVERY SYSTEM}

Work under Kropp at the University of Oklahoma has extensively evaluated porcine small-intestinal submucosa (SIS) as a scaffold for bladder regeneration. The use of unseeded natural scaffolds, for example SIS, for bladder tissue engineering has resulted in an inconsistent quality of bladder regeneration. Inconsistent regeneration using SIS has been attributed to the heterogeneous nature of the scaffold with physical properties varying by several factors including the bowel segment of origin (14).

Permeability of the scaffolds is one physical feature that is known to be inconsistent and may be a source of unpredictable regeneration; therefore, work in this lab has turned to using NPs as a means of altering scaffold permeability and simultaneous delivery of pro-regenerative compounds. Mondalek et al. (15) investigated the use of PLGA NPs to alter the permeability of SIS scaffolds. Preliminary experimentation with standard size latex beads indicated that particles ranging from 200 to $500 \mathrm{~nm}$ would become imbedded in the SIS scaffold. Particles below this size range would pass through the graft and not become entrapped, and particles above this size range could not penetrate the scaffold. In an attempt to produce a scaffold with consistent physical properties, the authors treated SIS scaffolds with NPs of PLGA. The SIS scaffolds were washed with a solution containing PLGA NPs ranging in size from 162 to $306 \mathrm{~nm}$. Scanning electron microscopy demonstrated uptake of the NPs within the SIS scaffold (Fig. 4). The modified scaffolds demonstrated a significant decrease in the permeability to urea when compared with unmodified scaffolds. Endothelial cells were cultured on both unmodified scaffold and PLGA modified scaffold with the modified SIS demonstrating a significantly higher concentration of cells by d 7 of culture.

The most recent research in Kropp's lab has investigated the use of PLGA NPs as a means of introducing proregenera- 

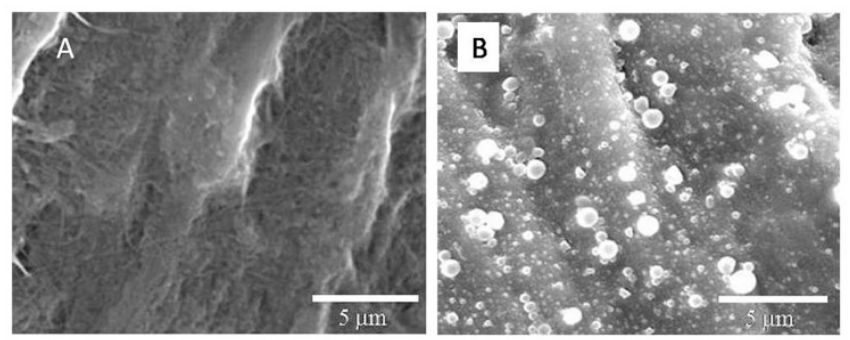

Figure 4. Representative scanning electron micrographs of standard SIS $(A)$ and SIS treated with PLGA NPS $(B)$. Note NPs both on the surface of and within the SIS scaffold. (Photos courtesy of F. Mondalek).

tive compounds into SIS scaffolds (16). One such compound is hyaluronic acid (HA), which has been recognized for its ability to promote scarless wound healing in part due to its angiogenic properties (17). Negatively charged HA was successfully bound to the external surface of the positively charged PLGA NPs. Physical characterization confirmed the presence of HA on the NPs by an increase in NP size and a change in the zeta potential of the NPs. SIS was then treated with a solution of HA-NPs and the presence of HA-NPs within the scaffold was confirmed via electron microscopy. To determine the rate of diffusion of HA from the scaffolds, the scaffolds were placed in PBS at $37^{\circ} \mathrm{C}$. Aliquots were collected over time and ELISA performed on the collected specimens demonstrated that the bound HA was released at a constant rate per time so that by $28 \mathrm{~d}$ more than $80 \%$ of the bound HA had been released.

Biocompatibility of the HA-NP SIS was assessed by culturing endothelial cells on both modified and control scaffolds. Genomic DNA was assayed after $7 \mathrm{~d}$ of culture and demonstrated statistically elevated cell growth on the HA-NP SIS. The angiogenic effect of HA-NP SIS was assessed using a chorionic amniotic membrane assay. Small sections of control SIS, NP SIS, and HA-NP SIS were placed on viable chicken embryos near a large blood vessel. After $5 \mathrm{~d}$ of incubation, the embryos were visually inspected and the number of new blood vessels was quantitated. Both NP SIS and HA-NP SIS demonstrated a statistically higher number of blood vessels when compared with unmodified SIS. The HA-NP SIS demonstrated the highest number of vessels overall though the difference between HA-NP SIS and NP SIS was not significant. HA-NP SIS was subsequently used in a dog model of bladder regeneration. Following $10 \mathrm{wk}$ of regeneration immunohistochemical staining using CD-31 antibodies demonstrated a higher density of new blood vessels in the regenerated bladder for dogs whose bladders were augmented with HA-NP SIS compared with unmodified SIS.

The earlier studies highlight a unique use of nanoscale polymers. The specific physical properties of the NPs allowed for uptake of the NPs into the natural scaffold. The NPs not only affected a potentially favorable change in a physical characteristic of the scaffold but also allowed for the delivery of a proregenerative compound. As research continues into this technology, additional growth factors can be combined with the NPs in an attempt to promote optimal regeneration.

\section{CONCLUSION}

Successful bladder tissue engineering will have a direct impact on pediatric urology; however, the ideal protocol for bladder tissue engineering has yet to be identified. All existing protocols rely on either natural or synthetic scaffolds as basis for tissue regeneration. Nanotechnology is currently being explored as a means to improve interaction between these scaffolds and the newly developing tissue. It is recognized that cell-substrate interactions occur on the nanoscale level and recent work with selective degradation and peptide self assembly has successfully introduced nanoscale features on existing synthetic scaffolds. These techniques provide evidence of improved cell growth and extracellular matrix deposition. Electrospinning has been used to create both synthetic and natural scaffolds based on the building block of nanoscale fibers. This technique has proven adaptable and allows for finely tuned creation of de novo scaffolds. NPs of PLGA have recently been used to enhance the physical properties of SIS. The same NPs also allow for the delivery of HA, which has demonstrated evidence of improved cell growth and angiogenesis in preclinical studies. It is likely that these techniques or the improved understanding of cell-substrate interactions gleaned from researching these techniques will be a part of the relevant bladder engineering protocols of the future.

\section{REFERENCES}

1. Harrington DA, Sharma AK, Erickson BA, Cheng EY 2008 Bladder tissue engineering through nanotechnology. World J Urol 26:315-322

2. Hasirci V, Vrana E, Zorlutuna P, Ndreu A, Yilgor P, Basmanav FB, Aydin E 2006 Nanobiomaterials: a review of the existing science and technology, and new approaches. J Biomater Sci Polym Ed 17:1241-1268

3. Thapa A, Webster TJ, Haberstroh KM 2003 Polymers with nano-dimensional surface features enhance bladder smooth muscle cell adhesion. J Biomed Mater Res A $67: 1374-1383$

4. Thapa A, Miller DC, Webster TJ, Haberstroh KM 2003 Nano-structured polymers enhance bladder smooth muscle cell function. Biomaterials 24:2915-2926

5. Pattison MA, Wurster S, Webster TJ, Haberstroh KM 2005 Three-dimensional, nano-structured PLGA scaffolds for bladder tissue replacement applications. Biomaterials 26:2491-2500

6. Miller DC, Haberstroh KM, Webster TJ 2005 Mechanism(s) of increased vascular cell adhesion on nanostructured poly(lactic-co-glycolic acid) films. J Biomed Mater Res A 73:476-484

7. Pattison M, Webster TJ, Leslie J, Kaefer M, Haberstroh KM 2007 Evaluating the in vitro and in vivo efficacy of nano-structured polymers for bladder tissue replacement applications. Macromol Biosci 7:690-700

8. Harrington DA, Cheng EY, Guler MO, Lee LK, Donovan JL, Claussen RC, Stupp SI 2006 Branched peptide-amphiphiles as self-assembling coatings for tissue engineering scaffolds. J Biomed Mater Res A 78:157-167

9. Pham QP, Sharma U, Mikos AG 2006 Electrospinning of polymeric nanofibers for tissue engineering applications: a review. Tissue Eng 12:1197-1211

10. Han D, Gouma PI 2006 Electrospun bioscaffolds that mimic the topology of extracellular matrix. Nanomedicine 2:37-41

11. Baker SC, Atkin N, Gunning PA, Granville N, Wilson K, Wilson D, Southgate J 2006 Characterisation of electrospun polystyrene scaffolds for three-dimensional in vitro biological studies. Biomaterials 27:3136-3146

12. McManus M, Boland E, Sell S, Bowen W, Koo H, Simpson D, Bowlin G 2007 Electrospun nanofibre fibrinogen for urinary tract tissue reconstruction. Biomed Mater 2:257-262

13. Stankus JJ, Freytes DO, Badylak SF, Wagner WR 2008 Hybrid nanofibrous scaffolds from electrospinning of a synthetic biodegradable elastomer and urinary bladder matrix. J Biomater Sci Polym Ed 19:635-652

14. Kropp BP, Cheng EY, Lin HK, Zhang Y 2004 Reliable and reproducible bladder regeneration using unseeded distal small intestinal submucosa. J Urol 172:1710-1713

15. Mondalek FG, Lawrence BJ, Kropp BP, Grady BP, Fung KM, Madihally SV, Lin HK 2008 The incorporation of poly(lactic-co-glycolic) acid nanoparticles into porcine small intestinal submucosa biomaterials. Biomaterials 29:1159-1166

16. Mondalek FG, Ashley RA, Roth CC, Kibar Y, Ihnat MA, Fung KM, Grady BP, Kropp BP, Lin HK. Enhanced angiongenesis of porcine small inestinal submucosa via hyaluronic acid-poly(lactide-co-glycolide) nanoparticles: from fabrication to pre-clinical validation. J Biomed Mater Res A, in press

17. Bullard KM, Longaker MT, Lorenz HP 2003 Fetal wound healing: current biology. World J Surg 27:54-61 